\title{
Anti-interleukin 1 treatment in secondary renal amyloidosis associated with autoinflammatory diseases
}

\author{
R Topaloglu, ED Batu², D Orhan³ ${ }^{3}$ S Ozen², N Besbas ${ }^{1}$ \\ From 8th International Congress of Familial Mediterranean Fever and Systemic Autoinflammatory Diseases \\ Dresden, Germany. 30 September - 3 October 2015
}

\begin{abstract}
Introduction
Amyloidosis represents a heterogeneous group of disorders characterized by extracellular deposition of autologous fibrillary proteins which impair normal organ function. Reactive AA type amyloidosis may complicate autoinflammatory diseases (AID).
\end{abstract}

\section{Objective}

To evaluate and compare the renal biopsy findings and clinical and laboratory parameters in patients with amyloidosis secondary to AID who have responded to the anti-interleukin 1 (IL1) treatment.

\section{Patients and methods}

Two children with systemic juvenile idiopathic arthritis and one with cryopyrin-associated periodic syndrome diagnosed as AA type amyloidosis were treated with anti-IL1 drugs and we have evaluated the course and management of these patients for a follow-up of median 56 (41-56) months. The renal biopsies at the time of diagnosis of amyloidosis and after the onset of anti-IL1 treatment were evaluated and compared according to the amyloid scoring and grading system based on the histopathological findings.

\section{Results}

The median age of AID onset was three years, while the patients were diagnosed to have amyloidosis at a median of 12 years of age. The patients previously used nonsteroidal anti-inflammatory drugs, corticosteroid, methotrexate, azathioprine, infliximab, and intravenous immunoglobulin treatments. After the diagnosis of amyloidosis, anakinra was started. All three responded to anakinra treatment;

'Hacettepe University Faculty of Medicine, Department of Pediatrics, Division of Nephrology, Ankara, Turkey

Full list of author information is available at the end of the article however, canakinumab was commenced in patient 3 since anakinra caused local cutaneous reaction at the site of drug administration. Proteinuria was improved in patients after anti-IL 1 treatment. Control renal biopsies were performed a median of three years later than the diagnosis of amyloidosis. At the renal biopsy level, we have seen that the renal amyloid prognostic score did not improve in patient 1 and progressed in patient 2 and 3 . The renal amyloid grade has also progressed in patient 2 .

\section{Conclusion}

To the best of our knowledge, this is the first series showing progression of renal tissue damage after the improvement of proteinuria with anti-IL 1 treatment in AID-associated amyloidosis. After the development of amyloidosis, it is crucial to control inflammation effectively and prevent further amyloid accumulation in patients with anti-inflammatory treatments such as antiIL1 drugs. However, new treatment strategies are needed to target the amyloid deposits for patients with severe organ involvement.

\section{Authors' details}

'Hacettepe University Faculty of Medicine, Department of Pediatrics, Division of Nephrology, Ankara, Turkey. ${ }^{2}$ Hacettepe University Faculty of Medicine,

Department of Pediatrics, Division of Rheumatology, Ankara, Turkey.

${ }^{3}$ Hacettepe University Faculty of Medicine, Department of Pediatric

Pathology, Ankara, Turkey.

Published: 28 September 2015

doi:10.1186/1546-0096-13-S1-P149

Cite this article as: Topaloglu et al: Anti-interleukin 1 treatment in secondary renal amyloidosis associated with autoinflammatory diseases. Pediatric Rheumatology 2015 13(Suppl 1):P149. 